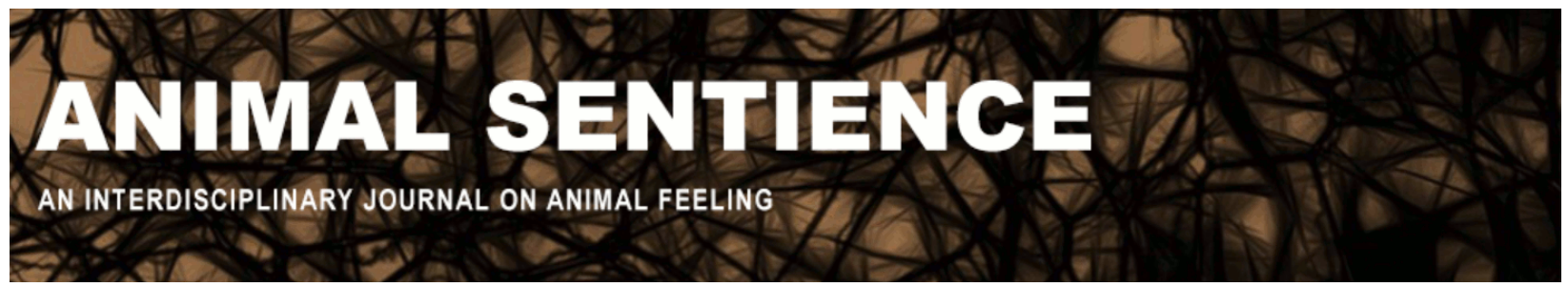

Mobbs, Dean (2018) What can the social emotions of dogs teach us about human emotions?. Animal Sentience 22(5)

DOI: $10.51291 / 2377-7478.1332$

Date of submission: 2018-05-22

Date of acceptance: 2018-05-26

(c)

This article has appeared in the journal Animal

Sentience, a peer-reviewed journal on animal

cognition and feeling. It has been made open access,

free for all, by WellBeing International and deposited

in the WBI Studies Repository. For more information,

please contact

wbisr-info@wellbeingintl.org.

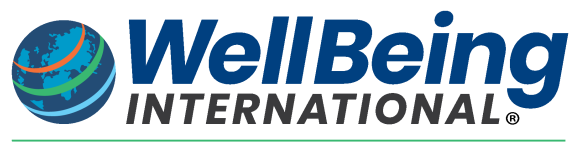

SOLUTIONS FOR PEOPLE, ANIMALS AND ENVIRONMENT 


\title{
What can the social emotions of dogs teach us about human emotions?
}

\author{
Commentary on Cook et al. on Dog Jealousy
}

\author{
Dean Mobbs \\ California Institute of Technology
}

\begin{abstract}
It has long been believed that social emotions such as guilt and jealousy are only expressed in humans. In the case of jealousy, its adaptive value has been linked to the prevention of sexual infidelity or fairness. So why would dogs feel jealousy? I suggest that understanding how social emotions have been bred into dogs can help us understand our own emotions, including their functionality - and potentially their mechanisms.
\end{abstract}

Dean Mobbs is a social and affective neuroscientist who works at the intersection of behavioral ecology, economics, emotion, and social psychology. He received his PhD from University College London and is now an assistant professor of cognitive neuroscience at the California Institute of

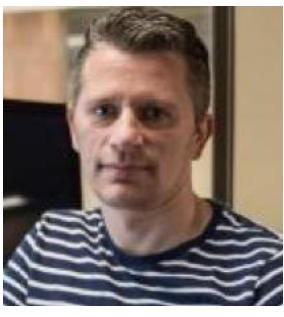
Technology. www.deanmobbslab.com

Canines have been domesticated by humans for over 10,000 years (Larson et al., 2012). In modern western society, they are the oldest and among the most popular of domesticated species (i.e., pets). Given the close, sometimes symbiotic, relationship between humans and dogs (canis familiaris), it is inevitable that humans are intrigued enough to study the neural circuits in this species. What makes dogs interesting is their amenability to human artificial selection (i.e., domestication), which has led to entirely new branches of taxa. These branches were bred for a variety of usual human purposes, including hunting, protection, and in most modern cases, companionship. The utility of dogs differs across strains from the great intelligence of the Border Collie to those that are bred for health or even aesthetic purposes (i.e., the Chihuahua). Across most species of domesticated dog, however, it is their remarkable capacity to exhibit human-like social emotions that has led many to believe that they may have evolved a set of neural circuits resembling the social brain circuits observed in humans.

Longitudinal work in Russia has shown that foxes can be artificially molded to be cute (i.e., pedomorphosis) and obedient or nasty-looking and aggressive (Trut, 1999). The engineering of these foxes occurred over very short periods of time (i.e., 40 years), and it is clear that the emotional style of the animal can also be molded in a way that is adaptive to humans. Recent research has also shown that domesticated canines have developed facial muscles so that they can express emotion, unlike their wolf ancestors (Waller et al., 2013). The domesticated dog is also very loyal and obedient and listens upon instruction. This is matched only by higher primates. But whereas primates evolved similar traits via natural selection, the canine species has been socially engineered by humans. It is this engineering of social emotions that gives us insight into 
the functions of our own social emotions. Panksepp and Biven (2012) go one step further by stating that "we do not believe that one can understand human emotional feeling without understanding those of fellow animals" (p. 71).

The current theoretical landscape of affective neuroscience. The word "emotion" is an umbrella term for a set of basic and higher-order social emotions. The basic emotions, which include fear, anger, disgust, sadness, surprise and joy, are believed to occur in many higher mammals (e.g., primates, canines, elephants, and dolphins). On the other hand, emotions such as guilt, awe and jealousy are believed to be a combination of these basic emotions and part of a rich palette of social emotions that are thought to occur only in humans. Ask any dog caregiver, however, and they will ardently tell you that their dog exhibits these same social emotions. How do these anecdotal observations fit with contemporary theories of emotion? In one camp, mainly captured by Barrett's (2017) Conceptual Act Theory, emotions are not universal; they emerge from the combination of external and internal sensations evolved through various distributed brain circuits that create emotional experiences and perceptions. In contrast, Ekman and Panksepp (2011) argue that a set of basic universal and domain-specific emotions is hard-wired in the brain (e.g., fear and rage circuits). Research by Cook and colleagues (2018) seems to support the latter in showing that in dogs, complex social emotions such as jealousy may involve the same neural structures as in humans (Takahashi et al., 2006), which suggests that they may be not only universal, but may also extend across species. The question is still open, however, as to whether dogs experience social emotions in the same way we do. Do they, like humans, have tertiary emotional processes that involve the cortical circuits of metacognition, rumination and consciousness? The answer could support other theories, including Conceptual Act Theory.

Feelings vs. automata. Adolphs's (2017) review asked whether dogs have conscious emotions. The answer is controversial, as advocates of the "dogs have feelings" camp (e.g., Safina 2016) have mostly leaned on behavioral observations that are biased by anthropomorphism and restricted by the inability to gain subjective reports. This is one of the central arguments of LeDoux's (2012) Survival Circuits Theory, in which only overt behaviors can be used to examine survival circuits (including social emotions) in animals. Most controversial is the notion that fear is a conscious experience and can only be studied in humans. What is interesting is that in several species, imaging tools such as $\mathrm{FMRI}$ have allowed us to see inside the animal brain during the evocation of a variety of emotions (e.g., in primates: Oler et al., 2010). Conditions that evoke a sense of danger activate threat circuits (see Mobbs, in press), such as the amygdala; reward states induce dopaminergic activity in the striatum in a growing list of species. The work of Cook and colleagues (2018) extends this research to canines, yet we are still left with the question of whether dogs feel emotions as we do or they are just emotional automata. The evidence is not there yet, but it certainly reveals some conserved capacity in dogs to express emotions, involving circuits similar to those in the human brain. An interesting question is whether we should see automatisms and conscious feelings as a continuous dimension rather than as two discrete categories.

What do dogs tell us about our emotions? Dogs mirror our social emotions because they have been engineered to do so. If we agree with this statement, then we can ask a series of questions: 
(i) Are expressions of emotion used as tools of communication or manipulation? Any parent will tell you that a child will learn to use expressions of emotion instrumentally to acquire a goal (e.g., ice cream). The emerging consensus is that facial expressions of emotional states are used as core social communication tools (Jack and Schyns, 2015). As mentioned, domesticated dogs have a unique set of facial muscles that mirror those of humans - muscles not observed in wolves - leading to the conclusion that these muscles have been artificially selected to mimic human facial expression. This theory, however, is difficult to prove and would require comparisons between different species of dog.

(ii) How does scientific work on dogs influence theories of emotions in humans? LeDoux's Survival-Circuits Theory still stands because we cannot ask dogs how they feel. Yet the enriched facial expressions and overlapping emotional circuits in dogs support the idea that they may feel emotions as we do. Dogs certainly exhibit sustained emotions (e.g., shivering excitement), yet the question is still open whether they have the conscious ability to process feelings as we do (I doubt this). Panksepp's notion of hard-wired circuits, which are observed in rodents and potentially now dogs, seems plausible, but whether jealousy is just an extension of the rage circuit or rather of tertiary cognitive circuits is not known. In her book, Barrett (2017) asks: "Is a growling dog angry"? Conceptual Act Theory suggests that the social emotions of dogs are the expression of their own emotional concepts but that dogs probably don't have a concept of jealousy (although she seems open to this, given the unique domestication of dogs).

(iii) Are our own emotions socially engineered? I think they are engineered, at least partially. Developmental theories suggest that social learning occurs early in life and that a parent's expression of emotion will result in the child's learning that same emotional expression vicariously (Eisenberg, Cumberland and Spinrad, 1998). The expression of emotions may differ across cultures (Tsai, Knutson and Fung, 2006), supporting the idea that culture engineers our emotions. Much like our parents, we have created an environment where we engineer the emotions of other species.

In conclusion, the behavioral and neuroscientific work on dogs raises questions about why we have social emotions: what is their functionality? The work on dogs teaches us that emotions have instrumental value in social communication, with jealousy providing the observer with a signal of discontent or aggression. This research also reveals that emotions can be socially engineered and that humans and dogs have a common set of social neural circuits. Although subjective verbal reports cannot be obtained from dogs, the work of Cook and colleagues (2018) suggests that the behavioral and neural manifestations of canine jealousy overlap with those of humans, taking us closer to understanding whether dogs have human-like feelings. 


\section{References}

Adolphs, R. (2017) Dogs consciously experience emotions: The question is, which?. Animal Sentience 14(2).

Cook, P., Prichard, A., Spivak, M., and Berns, G. S. (2018) Jealousy in dogs? Evidence from brain imaging. Animal Sentience 22(1).

Barrett, L. F. (2017) How emotions are made: The secret life of the brain. New York: Houghton Mifflin Harcourt.

Eisenberg, N., Cumberland, A., and Spinrad, T. L. (1998) Parental socialization of emotion. Psychological Inquiry, 9(4), 241-273.

Jack, R. E., and Schyns, P. G. (2015) The human face as a dynamic tool for social communication. Current Biology, 25(14), R621-R634.

Larson, G., Karlson, E. K., and Perri, A (2012) Rethinking dog domestication by integrating genetics, archeology, and biogeography. PNAS, 109(23), 8878-8883.

LeDoux, J. (2012) Rethinking the emotional brain. Neuron, 73, 653-676.

Mobbs, D. (In Press) The ethological deconstruction of fear(s). Current Opinion in Behavioral Sciences.

Oler, J. A., Fox, A. S., Shelton, S. E., Rogers, J., Dyer, T. D., Davidson, R. J., Shelledy, W., Oakes, T. R., Blangero J., and Kalin, N. H. (2010) Amygdalar and hippocampal substrates of anxious temperament differ in their heritability. Nature, 466(7308), 864.

Panksepp, J. (2011). The basic emotional circuits of mammalian brains: Do animals have affective lives? Neuroscience \& Biobehavioral Reviews, 35, 1791-1804.

Panksepp, J., and Biven, L. (2012) The archaeology of the mind. W.W. Norton and Company: New York.

Safina, C. (2016) Animals think and feel: Précis of Beyond words: What animals think and feel (Safina 2015). Animal Sentience 2(1).

Takahashi., H, Matsuura., M, Yahata, N., Koeda, M., Suhara, T., and Okubo, Y. (2006) Men and women show distinct brain activations during imagery of sexual and emotional infidelity. Neuroimage, Sep.;32(3), 1299-307.

Trut, L. N. (1999). Early canid domestication: The farm-fox experiment: Foxes bred for tamability in a 40-year experiment exhibit remarkable transformations that suggest an interplay between behavioral genetics and development. American Scientist, 87(2), 160-169.

Tsai, J. L., Knutson, B., and Fung, H. H. (2006). Cultural variation in affect valuation. Journal of Personality and Social Psychology, 90(2), 288-307.

Waller, B. M., Peirce, K., Caeiro, C. C., Scheider, L., Burrows, A. M., McCune, S., and Kaminski, J. (2013). Paedomorphic facial expressions give dogs a selective advantage. PLoS One, 8(12), e82686. 


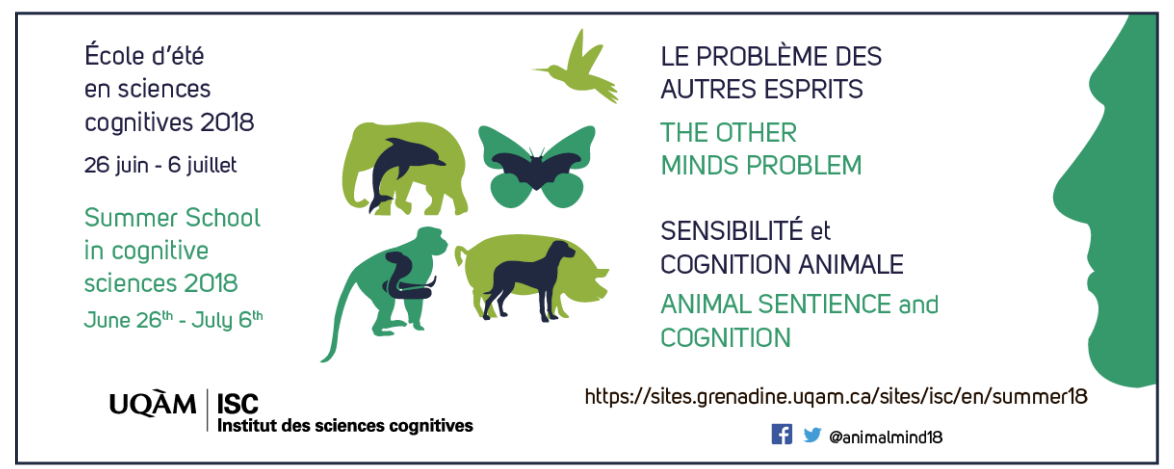

ISC 2018 Summer School in Cognitive Sciences June 26 - July 6, 2018 Montreal (Canada) The Other Minds
Problem: Animal
Sentience and Cognition

Overview. Since Descartes, philosophers know there is no way to know for sure what — or whether — others feel (not even if they tell you). Science, however, is not about certainty but about probability and evidence. The 7.5 billion individual members of the human species can tell us what they are feeling. But there are 9 million other species on the planet (20 quintillion individuals), from elephants to jellyfish, with which humans share biological and cognitive ancestry, but not one other species can speak: Which of them can feel - and what do they feel? Their human spokespersons - the comparative psychologists, ethologists, evolutionists, and cognitive neurobiologists who are the world's leading experts in "mindreading" other species - will provide a sweeping panorama of what it feels like to be an elephant, ape, whale, cow, pig, dog, chicken, bat, fish, lizard, lobster, snail: This growing body of facts about nonhuman sentience has profound implications not only for our understanding of human cognition, but for our treatment of other sentient species.

|Gregory Berns: Decoding the Dog's Mind with Awake Neuroimaging

Gordon Burghardt: Probing the Umwelt of Reptiles

Jon Sakata: Audience Effects on Communication Signals

PANEL 1: Reptiles, Birds and Mammals

WORKSHOP 1: Kristin Andrews: The "Other" Problems:

Mind, Behavior, and Agency

Sarah Brosnan: $\underline{\text { How Do Primates Feel About Their Social }}$

Partners?

Alexander Ophir: The Cognitive Ecology of Monogamy Michael Hendricks: Integrating Action and Perception in a Small Nervous System

PANEL 2: Primates, Voles and Worms

WORKSHOP 2: Jonathan Birch: Animal Sentience and the

Precautionary Principle

Malcolm Maclver: How Sentience Changed After Fish

Invaded Land 385 Million Years Ago

Sarah Woolley: Neural Mechanisms of Preference in Female

Songbird

Simon Reader: Animal Social Learning: Implications for

Understanding Others

PANEL 3: Sea to Land to Air

WORKSHOP 3: Steven M. Wise: Nonhuman Personhood

Tomoko Ohyama: Action Selection in a Small Brain

(Drosophila Maggot)

Mike Ryan: "Crazy Love": Nonlinearity and Irrationality in Mate Choice

Louis Lefebvre: Animal Innovation: From Ecology to

Neurotransmitters

PANEL 4: Maggots, Frogs and Birds: Flexibility Evolving SPECIAL EVENT: Mario Cyr: Polar Bears

Colin Chapman: Why Do We Want to Think People Are Different?

Vladimir Pradosudov: Chickadee Spatial Cognition Jonathan Balcombe: The Sentient World of Fishes

PANEL 5: Similarities and Differences

WORKSHOP 5 (part 1): Gary Comstock: A Cow's Concept of Her Future

WORKSHOP 5 (part 2): Jean-Jacques Kona-Boun: Physical

and Mental Risks to Cattle and Horses in Rodeos
Joshua Plotnik: Thoughtful Trunks: Application of Elephant Cognition for Elephant Conservation

Lori Marino: Who Are Dolphins?

Larry Young: The Neurobiology of Social Bonding, Empathy and Social Loss in Monogamous Voles

Panel 6: Mammals All, Great and Small

WORKSHOP 6: Lori Marino: The Inconvenient Truth About

Thinking Chickens

Andrew Adamatzky: Slime Mould: Cognition Through

Computation

Frantisek Baluska \& Stefano Mancuso: What a Plant Knows and Perceives

Arthur Reber: $\mathrm{A}$ Novel Theory of the Origin of Mind:

Conversations With a Caterpillar and a Bacterium

PANEL 7: Microbes, Molds and Plants

WORKSHOP 7: Suzanne Held \& Michael Mendl: Pig

Cognition and Why It Matters

James Simmons: What Is It Like To Be A Bat?

Debbie Kelly: Spatial Cognition in Food-Storing

Steve Phelps: Social Cognition Across Species

PANEL 8: Social Space

WORKSHOP 8: To be announced

Lars Chittka: The Mind of the Bee

Reuven Dukas: Insect Emotions: Mechanisms and

Evolutionary Biology

Adam Shriver: Do Human Lesion Studies Tell Us the Cortex

is Required for Pain Experiences?

PANEL 9: The Invertebrate Mind

WORKSHOP 9: Delcianna Winders: Nonhuman Animals in

Sport and Entertainment

Carel ten Cate: Avian Capacity for Categorization and

Abstraction

Jennifer Mather: Do Squid Have a Sense of Self?

Steve Chang: Neurobiology of Monkeys Thinking About Other Monkeys

PANEL 10: Others in Mind

WORKSHOP 10: The Legal Status of Sentient Nonhuman

Species 